\title{
On the exact and numerical solutions to a nonlinear model arising in mathematical biology
}

\author{
Asif Yokus ${ }^{1}$, Tukur Abdulkadir Sulaiman, Haci Mehmet Baskonus ${ }^{4}$, and Sibel Pasali \\ Atmaca $^{5, *}$ \\ ${ }^{1}$ Department of Actuary, Firat University, Elazig, Turkey \\ ${ }^{2}$ Department of Mathematics, Firat University, Elazig, Turkey \\ ${ }^{3}$ Department of Mathematics, Federal University, Dutse, Jigawa, Nigeria \\ ${ }^{4}$ Department of Computer Engineering, Munzur University, Tunceli, Turkey \\ ${ }^{5}$ Mugla Sitki Kocman University, Mugla, Turkey
}

\begin{abstract}
This study acquires the exact and numerical approximations of a reaction-convection-diffusion equation arising in mathematical biology namely; Murry equation through its analytical solutions obtained by using a mathematical approach; the modified $\exp (-\Psi(\eta))$-expansion function method. We successfully obtained the kink-type and singular soliton solutions with the hyperbolic function structure to this equation. We performed the numerical simulations (3D and 2D) of the obtained analytical solutions under suitable values of parameters. We obtained the approximate numerical and exact solutions to this equation by utilizing the finite forward difference scheme by taking one of the obtained analytical solutions into consideration. We investigate the stability of the finite forward difference method with the equation through the Fourier-Von Neumann analysis. We present the $L_{2}$ and $L_{\infty}$ error norms of the approximations. The numerical and exact approximations are compared and the comparison is supported by a graphic plot. All the computations and the graphics plots in this study are carried out with help of the Matlab and Wolfram Mathematica softwares. Finally, we submit a comprehensive conclusion to this study.
\end{abstract}

\section{Introduction}

Partial differential equations (PDEs) serve as the tremendous tools that bond the realistic problems and mathematical theories. PDEs describe various complex real life aspects arising in the various fields of science, such as; mathematical biology, chemistry, mathematical physics and son. It is sometimes difficult to investigate the solutions of these type of models, more especially in their nonlinear forms. Various mathematical approaches have been developed and invested to solve such kind of models [1-18].

The aim of this research is to acquire the exact and numerical approximations of a nonlinear model that arises in mathematical biology through its analytical solutions

\footnotetext{
*Corresponding Author: sibela@mu.edu.tr
} 
obtained by using the modified exp $(-\Omega(\eta)$ )-expansion function method (MEFM) $[19,20]$. The finite forward difference method [21] is going to be used for the exact and numerical solutions approximation. Mathematical biology is one of the interesting and most exciting modern application of mathematics.

This study considers a particular case of reaction-convection-diffusion equations with a convection term given by [22]:

$$
u_{t}=\left(A(u) u_{x}\right)_{x}+B(u) u_{x}+C(u),
$$

where $A(u), B(u)$ and $C(u)$ are arbitrary smooth functions.

Eq. (1) stands for the generalized form of various second order nonlinear evolution equations that describe various nonlinear processes in biology [23, 24].

The governing equation: The Murry equation which is the particular case of Eq. (1) and considered as a generalization of the Fisher and Burgers equations is given by $[22,25]$ :

$$
u_{t}-u_{x x}-a u u_{x}-b u+c u^{2}=0,
$$

where $a, b$ and $c$ are nonzero constants.

\section{The Analysis of MEFM}

In this section, we present the description of the MEFM . The MEFM is designed by improving the popularly known exp $(-\Psi(\eta))$-expansion function method. To explore the search for the new solutions of any given nonlinear partial differential equation (Eq. (3)), we follow the following steps:

$$
P\left(u, u_{x}, u_{x} u^{2}, u_{x x}, u_{x x t}, \ldots\right),
$$

where $u=u(x, t)$ is unknown function, $P$ is a polynomial in $u(x, t)$ and its derivatives and the subscripts stand for the partial derivatives.

Step 1: Consider the wave transformation given as follows

$$
u(x, t)=U(\eta), \quad \eta=k(x-c t),
$$

using Eq. (4) on Eq. (3), gives the following nonlinear ordinary differential equation (NODE):

$$
D\left(U, U^{2}, U^{\prime}, U^{\prime \prime}, \ldots\right),
$$

where $D$ is a polynomial of $U$ and its derivatives and the superscripts stand for the ordinary derivatives of $U$ with respect to $\eta$.

Step 2: Assuming that the wave solutions of Eq. (5) can be written in the following form:

$$
U(\eta)=\frac{\sum_{i=0}^{N} A_{i}\left[e^{-\Psi(\eta)}\right]^{i}}{\sum_{j=0}^{M} B_{j}\left[e^{-\Psi(\eta)}\right]^{j}}=\frac{A_{0}+A_{1} e^{-\Psi}+\ldots+A_{N} e^{-N \Psi}}{B_{0}+B_{1} e^{-\Psi}+\ldots+B_{M} e^{-M \Psi}},
$$


where $A_{i}, B_{j},(0 \leq i \leq N, 0 \leq j \leq M)$ are constants to be found later, such that $A_{N} \neq o, B_{M} \neq o$, and $\Psi=\Psi(\eta)$ simplifies the following ODE:

$$
\Psi^{\prime}(\eta)=e^{-\Psi(\eta)}+\mu e^{\Psi(\eta)}+\lambda .
$$

Eq.(7) has the following families of solutions [26-28]:

Family 1: When $\mu \neq 0, \lambda^{2}-4 \mu>0$,

$$
\Psi(\eta)=\ln \left(\frac{-\sqrt{\lambda^{2}-4 \mu}}{2 \mu} \tanh \left(\frac{\sqrt{\lambda^{2}-4 \mu}}{2}(\eta+E)\right)-\frac{\lambda}{2 \mu}\right) .
$$

Family 2: When $\mu \neq 0, \lambda^{2}-4 \mu<0$,

$$
\Psi(\eta)=\ln \left(\frac{\sqrt{-\lambda^{2}+4 \mu}}{2 \mu} \tan \left(\frac{\sqrt{-\lambda^{2}+4 \mu}}{2}(\eta+E)\right)-\frac{\lambda}{2 \mu}\right) .
$$

Family 3: When $\mu=0, \lambda \neq 0$ and $\lambda^{2}-4 \mu>0$,

$$
\Psi(\eta)=-\ln \left(\frac{\lambda}{e^{\lambda(\eta+E)}-1}\right)
$$

Family 4: When $\mu \neq 0, \lambda \neq 0$ and $\lambda^{2}-4 \mu=0$,

$$
\Psi(\eta)=\ln \left(-\frac{2 \lambda(\eta+E)+4}{\lambda^{2}(\eta+E)}\right) .
$$

Family 5: When $\mu=0, \lambda=0$ and $\lambda^{2}-4 \mu=0$,

$$
\Psi(\eta)=\ln (\eta+E)
$$

where $A_{i}, B_{j},(0 \leq i \leq N, 0 \leq j \leq M), E, \lambda, \mu$ are coefficients to be found later, and $M, N$ are positive integers that can be obtained by using the balancing technique.

Step 3: Putting Eq. (6) and its derivatives along with the Eq. (7) into Eq. (5) and solving, we find an equation involving polynomial of $e^{-\Psi(\eta)}$. We extract system of equations from that polynomial of $e^{-\Psi(\eta)}$ by summing all the terms of the same power and equating each summation to zero. To find the new solutions of (3), we simplify the system of equations with aid of the Wolfram Mathematica 11 to find the values of the various coefficients $A_{i}, B_{j},(0 \leq i \leq N, 0 \leq j \leq M), E, \lambda, \mu$. Putting the obtained values of the coefficients along with one of Eqs. (8-12) into Eq. (6), yields new solution to (3).

\section{The Analysis of FDM}

Presenting the finite forward difference method needs definitions of some notations given as

1. $\Delta x$ stands for the spatial step

2. $\Delta t$ stands for the time step 
3. $x_{i}=a+i \Delta x, i=0,1,2, \ldots, N$ are coordinates points of the mesh and $N=\frac{b-a}{\Delta x}$, $t_{j}=j \Delta x, j=0,1,2, \ldots, M$ and $M=\frac{T}{\Delta t}$.

4. The functions $u(x, t)$ represents the values of the solutions at the grid points which are $u\left(x_{i}, t_{j}\right) \approx u_{i, j}$. the $u_{i, j}$ and $v_{i, j}$ stands for the numerical solutions of the exact values of $u(x, t)$ at the point $\left(x_{i}, t_{j}\right)$

We therefore present the difference operators as follows:

$$
\begin{gathered}
H_{t} u_{i, j}=u_{i, j+1}-u_{i, j}, \\
H_{x} u_{i, j}=u_{i+1, j}-u_{i, j}, \\
H_{x x} u_{i, j}=u_{i+1, j}-2 u_{i, j}+u_{i-1, j} .
\end{gathered}
$$

Hence, the partial derivatives can be approximated through the finite difference operators as:

$$
\begin{gathered}
\left.\frac{\partial u}{\partial t}\right|_{i, j}=\frac{H_{t} u_{i, j}}{\Delta t}+O((\Delta t)), \\
\left.\frac{\partial u}{\partial x}\right|_{i, j}=\frac{H_{x} u_{i, j}}{\Delta x}+O((\Delta x)), \\
\left.\frac{\partial^{2} u}{\partial x^{2}}\right|_{i, j}=\frac{H_{x x} u_{i, j}}{(\Delta x)^{2}}+O\left((\Delta x)^{2}\right),
\end{gathered}
$$

Eq. (2) is given in operators form as:

$$
\frac{H_{t} u_{i, j}}{\Delta t}-\frac{H_{x x} u_{i, j}}{(\Delta x)^{2}}-a u_{i, j} \frac{H_{x} u_{i, j}}{\Delta x}-b u_{i, j}+c u_{i, j}^{2}=0 .
$$

The following indexed form is produced by inserting Eq. (13-15) into Eq. (19)

$$
\begin{array}{r}
u_{i+1, j}=\frac{1}{(\Delta t)(\Delta x) u_{i, j}}\left(2(\Delta t) u_{i, j}-(\Delta t) u_{i-1, j}-(\Delta x)^{2} u_{i, j}+2 \sqrt{5}(\Delta t)(\Delta x)^{2} u_{i, j}\right. \\
\left.+(\Delta t)(\Delta x) u_{i, j}^{2}+(\Delta t)(\Delta x)^{2} u_{i, j}^{2}-(\Delta t) u_{i, j+1}+(\Delta x)^{2} u_{i, j+1}\right)
\end{array}
$$

\section{Von-Neumann Stability Analysis}

In this section, we examine the stability of the numerical scheme with the use the Eq. (2) by using the Von-Neumann stability analysis. We consider $\eta^{n}$ to be the amplication factor, and the growth factor of a typical Fourier mode to be

$$
u_{m}^{n}=\eta^{n} e^{i m \Phi}
$$

where $i=\sqrt{-1}$.

To investigate the stability of the numerical scheme with Eq. (2), we linearize the nonlinear terms $u u_{x}, u^{2}$ by making the quantity $\widehat{u}=u$ a local constant. Thus, the nonlinear terms in the equation becomes $\widehat{u} u_{x}$ and $\widehat{u}^{2}$ respectively. In this case Eq. (2) becomes

$$
u_{t}-u_{x x}-a \widehat{u} u_{x}-b u+c \widehat{u}^{2}=0 .
$$


Inserting Eq. (16), (17), (18) and the Fourier mode (21) into the recurrence relation (22), yields

$$
\eta=p+i q
$$

where

$$
\begin{array}{r}
p=\frac{1}{(\Delta t)-(\Delta x)^{2}}\left(2(\Delta t)+a \widehat{u}(\Delta x)(\Delta t)+(\Delta x)^{2}(c \widehat{u}(\Delta t)\right. \\
-b(\Delta t)-1)-(\Delta t)(a \widehat{u}(\Delta x)) \cos (\Phi))
\end{array}
$$

and

$$
q=\frac{1}{(\Delta t)-(\Delta x)^{2}}(1-a \widehat{u}(\Delta x))(\Delta t) \sin (\Phi) .
$$

The numerical scheme is said to be unconditionally stable as $|\eta| \leq 1$ [29].

\section{$5 \mathrm{~L}_{2}$ and $\mathrm{L}_{\infty}$ Error Norms}

For the test problem used in the present study, exact and numerical approximations of Eq. (2) have been investigated. To see how the exact and numerical approximations are close to each other we use $L_{2}$ and $L_{\infty}$ error norms. The error $L_{2}$ norms defined as [?]

$$
L_{2}=\left\|u^{\text {exact }}-u^{\text {numeric }}\right\|_{2}=\sqrt{h \sum_{j=0}^{N}\left|u_{j}^{\text {exact }}-u_{j}^{\text {numeric }}\right|^{2}},
$$

and $L_{\infty}$ error norm defined as

$$
L_{\infty}=\left\|u^{\text {exact }}-u^{\text {numeric }}\right\|_{\infty}={ }_{j}^{\text {Max }}\left|u_{j}^{\text {exact }}-u_{j}^{\text {numeric }}\right| .
$$

\section{Implementation of MEFM}

Consider the Murry equation given in Eq. (2).

With the following wave transformation; $u(x, t)=U(\eta), \eta=x-k t$, Eq. is transformed to the following NODE:

$$
U^{\prime \prime}+k U^{\prime}+a U U^{\prime}+b U-c U^{2}=0 .
$$

We obtain $N=M+2$ as relation between $M$ and $N$ under the terms $U^{\prime \prime}$ and $U^{2}$ by balancing technique.

Choosing $M=1$, gives $N=3$. Using $M=1, N=3$ and Eq. (6), yields the following equation:

$$
U(\eta)=\frac{A_{0}+A_{1} e^{-\Psi(\eta)}+A_{2} e^{-2 \Psi(\eta)}+A_{3} e^{-3 \Psi(\eta)}}{B_{0}+B_{1} e^{-\Psi(\eta)}} .
$$

We substitute Eq. (7), Eq. (27) with its first and second derivatives into Eq. (26) and obtain an equation in $e^{-\Psi(\eta)}$. We collect a set from algebraic equations from 
the obtained equation by setting each summation of the coefficients of $e^{-\Psi(\eta)}$ with the same power to zero. We simplify the set of equations with the aid of Wolfram Mathematica package and obtained the values of the coefficients involved in the equations in various cases. In order to obtain the wave solutions of Eq. (2), we insert the values of the coefficients into Eq. (27) along with suitable condition from family $1-5$.

\section{Case-1:}

$$
\begin{aligned}
& A_{0}=\frac{B_{0}}{a}\left(\lambda-\sqrt{\lambda^{2}-4 \mu}\right), A_{1}=\frac{1}{a}\left(2 B_{0}+\lambda B_{1}-B_{1} \sqrt{\lambda^{2}-4 \mu}\right), A_{2}=\frac{2 B_{1}}{a}, A_{3}=0, \\
& b=-\frac{2 c}{a} \sqrt{\lambda^{2}-4 \mu}, k=\sqrt{\lambda^{2}-4 \mu}-\frac{2 c}{a}
\end{aligned}
$$

When $\mu \neq 0, \lambda^{2}-4 \mu>0$, we get:

$$
u_{1.1}(x, t)=\frac{1}{a^{2}}\left(-a \sqrt{\lambda^{2}-4 \mu}+a\left(\lambda-\frac{4 \mu}{\left(\lambda+\sqrt{\lambda^{2}-4 \mu} \tanh \left[\Psi_{1.1}(x, t)\right]\right)}\right)\right)
$$

where $\Psi_{1.1}(x, t)=\frac{1}{2} \sqrt{\lambda^{2}-4 \mu}\left(E+x+\left(\frac{2 c}{a}-\sqrt{\lambda^{2}-4 \mu}\right) t\right)$.

When $\mu=0, \lambda \neq 0$ and $\lambda^{2}-4 \mu>0$, we get:

$$
u_{1.2}(x, t)=\frac{\lambda}{a}\left(\operatorname{coth}\left[\frac{1}{2} \lambda\left(E+x+\left(\frac{2 c}{a}-\lambda\right) t\right)\right]\right) .
$$
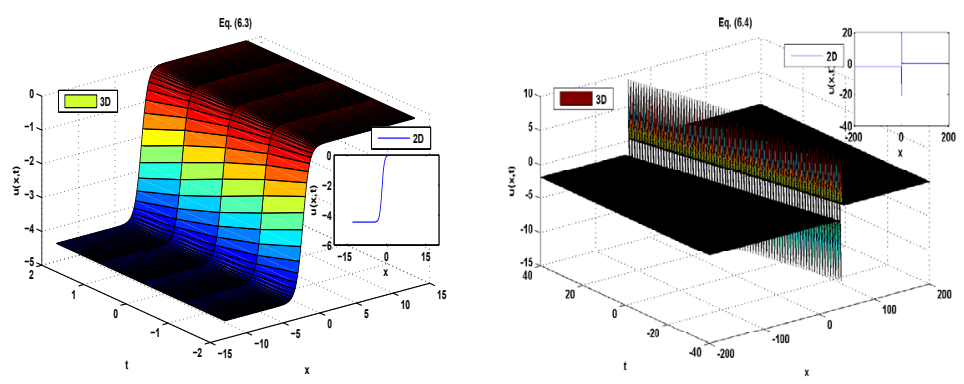

Figure 1. The kink-type and singular soliton surfaces of Eqs. (28) and (29) under the values $E=a=c=\mu=1, \lambda=3,(-15<x<15,-2<t<2$ for Eq. $(28)),(-200<x<200$, $-40<t<40$ for Eq. (29)) and $t=0.5$ for the $2 \mathrm{D}$ graphics.

\section{Case-2:}

$$
A_{0}=\frac{b}{2 c \sqrt{\lambda^{2}-4 \mu}}\left(B_{0}\left(\sqrt{\lambda^{2}-4 \mu}-\lambda\right)+2 \mu B_{1}\right), A_{2}=0, A_{3}=0, a=-\frac{2 c}{b} \sqrt{\lambda^{2}-4 \mu}
$$




\section{CMES-2018}

$$
A_{1}=\frac{b}{2 c \sqrt{\lambda^{2}-4 \mu}}\left(B_{1}\left(\sqrt{\lambda^{2}-4 \mu}+\lambda\right)+2 B_{0}\right), k=\frac{b+\lambda^{2}-4 \mu}{\sqrt{\lambda^{2}-4 \mu}},
$$

When $\mu \neq 0, \lambda^{2}-4 \mu>0$, we get:

$$
\begin{aligned}
& u_{2.1}(x, t)=-\left(2 c \sqrt{\lambda^{2}-4 \mu}\left(-2 \mu B_{1}+B_{0}\left(\lambda+\sqrt{\lambda^{2}-4 \mu} \tanh \left[\Psi_{2.1}(x, t)\right]\right)\right)\right)^{-1} \\
& \times\left(b\left(\left(\lambda\left(\sqrt{\lambda^{2}-4 \mu}-\lambda\right)+4 \mu\right) B_{0}-2 \mu B_{1} \sqrt{\lambda^{2}-4 \mu}\right)\right)\left(\tanh \left[\Psi_{2.1}(x, t)\right]-1\right),
\end{aligned}
$$

where $\Psi_{2.1}(x, t)=\frac{1}{2}\left((E+x) \sqrt{\lambda^{2}-4 \mu}-\left(b+\lambda^{2}-4 \mu\right) t\right)$.

When $\mu=0, \lambda \neq 0$ and $\lambda^{2}-4 \mu>0$, we get:

$$
u_{2.2}(x, t)=\frac{b\left(\lambda B_{1}-B_{0}\right)}{c \lambda}\left(\frac{B_{0}}{\lambda}\left(-1+e^{\lambda\left(E+x-\frac{\left(b+\lambda^{2}\right)}{\lambda} t\right)}\right)+B_{1}\right)^{-1} .
$$
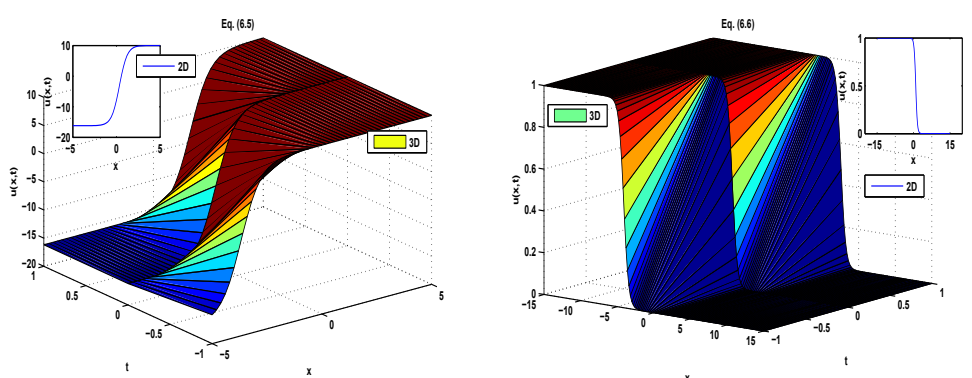

Figure 2. The kink-type surfaces of Eqs. (30) and (31) under the values $B_{0}=B_{1}=E=$ $b=c=1, \lambda=3,(-5<x<5,-1<t<1$ for Eq. (30)), $(-15<x<15,-1<t<1$ for Eq. (31)) and $t=0.5$ for the 2D graphics.

\section{Exact and Numerical Approximations}

In this section, we utilize the numerical scheme in obtaining the exact and numerical approximations to Eq. (2) by considering Eq. (28). Inserting $E=B_{1}=a=c=\mu=$ 1 and $\lambda=3$ into Eq. (28), yields the following special exact solution:

$$
u(x, t)=3-\sqrt{5}-\frac{4}{3+\sqrt{5} \tanh \left[\frac{1}{2} \sqrt{5}(1+(2+\sqrt{5}) t+x)\right]},
$$

at $t=0$, Eq. (32) becomes

$$
u_{0}(x)=3-\sqrt{5}-\frac{4}{3+\sqrt{5} \tanh \left[\frac{1}{2} \sqrt{5}(1+x)\right]} .
$$


Inserting $(\Delta x)=(\Delta t)=0.0001$ into Eq. (20), yields the following indexed form:

$$
\begin{array}{r}
u_{i+1, j}=\frac{1.000 \times 10^{8}}{u_{i, j}}\left(-0.0001 u_{i-1, j}+0.00019999 u_{i, j}\right. \\
\left.+1.0001 \times 10^{-8} u_{i, j}^{2}-0.00009999 u_{i, j+1}\right) .
\end{array}
$$

$L_{2}$ and $L_{\infty}$ errors norm table

Table 1.

\begin{tabular}{lcccc}
$(\Delta x)$ & $(\Delta t)$ & Numerical solution & Exact Solution & Eror \\
\hline 0 & 0.01 & -0.0686494 & -0.0686681 & $1.86873 \times 10^{-5}$ \\
0.01 & 0.01 & -0.0686343 & -0.0686530 & $1.86833 \times 10^{-5}$ \\
0.02 & 0.01 & -0.0686192 & -0.0686379 & $1.86792 \times 10^{-5}$ \\
0.03 & 0.01 & -0.0686041 & -0.0686228 & $1.86752 \times 10^{-5}$ \\
0.04 & 0.01 & -0.0685890 & -0.0686077 & $1.86712 \times 10^{-5}$ \\
0.05 & 0.01 & -0.0685739 & -0.0685926 & $1.86671 \times 10^{-5}$ \\
0.06 & 0.01 & -0.0685588 & -0.0685775 & $1.86631 \times 10^{-5}$ \\
\hline
\end{tabular}

Table 1: Numerical, exact approximations and absolute errors of Eq. (2) by considering Eq. (28), under the value $\Delta(x)=0.0001$ and $0 \leq x \leq 1$.

Table 2.

\begin{tabular}{lcc}
$\Delta(x)=\Delta(t)$ & $L_{2}$ & $L_{\infty}$ \\
\hline 0.1 & $1.0073 \times 10^{-2}$ & $1.9158 \times 10^{-2}$ \\
0.01 & $1.0167 \times 10^{-2}$ & $1.9158 \times 10^{-2}$ \\
0.001 & $5.3000 \times 10^{-5}$ & $1.8690 \times 10^{-4}$ \\
0.0001 & $8.8763 \times 10^{-6}$ & $1.8687 \times 10^{-5}$ \\
\hline
\end{tabular}

Figure 3. Numerical and exact approximations of Eq. (2) by considering Eq. (28).

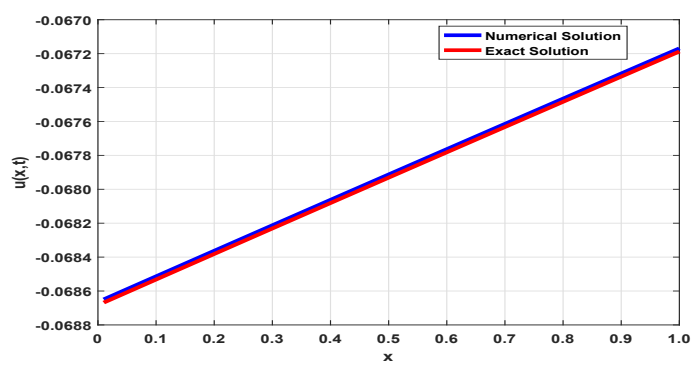

Fig. 3 illustrates the physical properties of the exact and numerical approximations, and it shows that the exact values are almost close to the numerically calculated values. We also observed that from Tab. 1 the truncation errors depend on the choice of $\Delta x$ and $\Delta t$. As $\Delta x$ and $\Delta t$ approaching zero we can see that the truncation errors are getting smaller. Also from Tab. 2 as $\Delta x$ and $\Delta t$ approaching zero, we can see that the $L_{2}$ and $L_{\infty}$ errors norm is getting smaller which is also indicating how close the exact and numerical approximations are. Fig. 4 illustrates the physical features of the absolute errors. 
Figure 4. Absolute error graphic at $\Delta(x)=\Delta(t)=0.0001$.

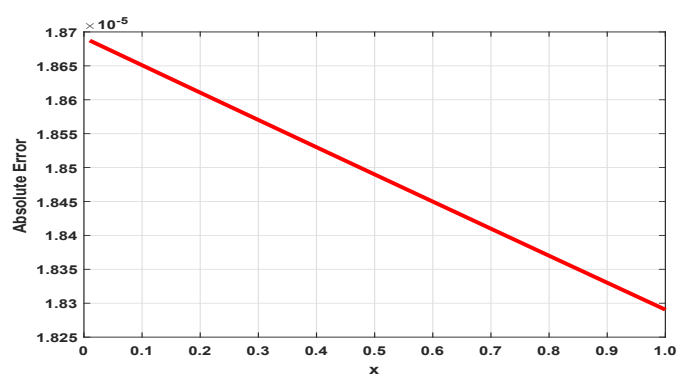

\section{Conclusions}

In this paper, with help of the Matlab and Wolfram Mathematica softwares, we investigate the solutions of a reaction-convection-diffusion equation, namely; Murry equation by using the modified $\exp (-\Psi(\eta))$-expansion function method. We successfully obtained some kink-type and singular soliton solutions with the hyperbolic function structure to this equation. We presented the interesting $2 \mathrm{D}$ and $3 \mathrm{D}$ graphics of all the obtained analytical solutions in this paper. Furthermore, through the obtained analytical solutions, we utilized the finite forward difference scheme in obtaining the exact and numerical approximations to this equation. We observed that the exact solutions is almost close to the calculated numerical solutions and we supported this comparison with a graphic plot (Fig. 3). We also observed that as $\Delta x$ and $\Delta t$ approaching zero, the truncation errors are getting smaller. The modified $\exp (-\Psi(\eta))$-expansion function method is efficient and simple mathematical tool that can be used to search for the analytical solutions of various nonlinear evolution equations. And, finite forward difference scheme is a powerful numerical method that can be used to find the approximate solutions to various nonlinear evolution equations through their analytical solutions.

\section{References}

[1] M. Matinfar, M. Eslami, S. Roshandel, Pramana-J Phys 85(4), 593-603 (2015)

[2] H. Bulut, T.A. Sulaiman and B. Demirdag, Nonlinear Dyn. 91 1985-1991 (2018)

[3] H. Bulut, H.A. Isik and T.A. Sulaiman, ITM Web of Conferences 13, 01019 (2017)

[4] H. Bulut, T.A. Sulaiman, H.M. Baskonus and T. Akturk, Opt Quant Electron 50, 134 (2018)

[5] A.S. Alofi, International Mathematical Forum 7(53), 2639-2649 (2012)

[6] H. Zhang, Communications in Nonlinear Science and Numerical Simulation 12(5), 627-635 (2007)

[7] B. Bülbül and M. Sezer, Journal of Applied Mathematics 2013, 691614 (2013)

[8] C. Cattani, T.A. Sulaiman, H.M. Baskonus and H. Bulut, 50, Opt Quant Electron 138 (2018)

[9] L. Zhu and S.L. Wu, International Journal of Biomathematics 10(6), 1750086 (2017)

[10] C. Cattani, T.A. Sulaiman, H.M. Baskonus and H. Bulut, Eur. Phys. J. Plus 133, 228 (2018) 
[11] H.M. Baskonus, T.A. Sulaiman and H. Bulut, Opt Quant Electron 50, 253 (2018)

[12] E. Fan and H. Zhang, Physics Letters A 246(5), 403-406 (1998)

[13] H. Bulut, T.A. Sulaiman, H.M. Baskonus, and A.A. Sandulyak, Optik 135, 327336 (2017)

[14] S. Guo, Y. Zhou, X Zhao, Applied Mathematics and Computation 215, 3214$3221(2010)$

[15] L. Mei, Y. Gao, and Z. Chen, Numerical Methods for Partial Differential Equations 31(6), 1875-1889 (2015)

[16] A. Esen and S. Kutluay, International Journal of Computer Mathematics 83(3), 319-330 (2006)

[17] S. Kutluay, Applied mathematics and computation 168(2), 1159-1168 (2005)

[18] A. Esen, B. Karaagac and O. Tasbozan, Appl Math Inf Sci Lett 4(1), 1-4 (2016)

[19] S. Duran, M. Askin and T.A. Sulaiman, IJOCTA 7(3), 240-247 (2017)

[20] A. Yokus, H.M. Baskonus, T.A. Sulaiman and H. Bulut, Numer Methods Partial Differential Eq. 34, 211-227 (2018)

[21] A. Yokus, T.A. Sulaiman and H. Bulut, Opt Quant Electron 50, 31 (2017)

[22] E.H.M. Zahran and M.M.A. Khater, Journal of Research in Applied Sciences 2(4) 101-107 (2015)

[23] J.D. Murry, Nonlinear Differential Equation Models in Biology, (Clarendon Press, Oxford, (1977)) 54-156

[24] J.D. Murry, Mathematical Biology, (Springer Berlin, (1989)) 45-123

[25] R.M. Cherniha, Symmetry in Nonlinear Mathematical Physics 1, 138-146 (1997)

[26] H.O. Roshid and M.A. Rahman, Results Phys 4, 150-155 (2014)

[27] A.E. Abdelrahman, E.H.M. Zahran and M.M.A. Khater, Int J Mod Nonlinear Theory Appl 4, 37-47 (2015)

[28] M.G. Hafez, M.N. Alam, and M.A. Akbar, World Appl Sci J 32, 2150-2155 (2014)

[29] A. Golbabai and E. Mohebianfar, Journal of Nonlinear Science and Applications, Comput Econ 49(2), 271-288 (2016) 